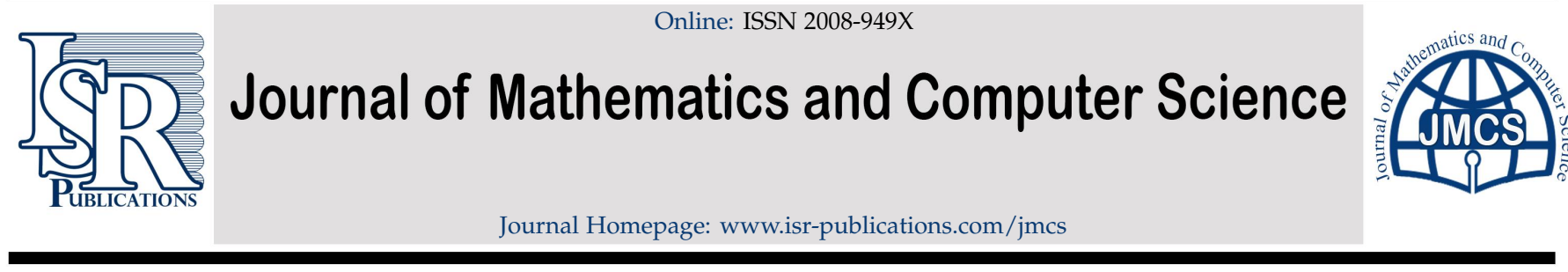

\title{
On bivariate Apostol-Fubini polynomials of higher order
}

\author{
Nestor G. Acala \\ Mathematics Department, Main Campus, Mindanao State University, Marawi City, Lanao Del Sur 9700, Philippines.
}

\begin{abstract}
Recently, some generalizations of the Apostol-Bernoulli polynomials, Apostol-Euler polynomials, and Apostol-Genocchi polynomials were introduced (see for instance [V. Kurt, Appl. Math. Sci., 3 (2009), 2757-2764], [Q.-M. Luo, Taiwanese J. Math., 10 (2006), 917-925], [Q.-M. Luo, H. M. Srivastava, J. Math. Anal. Appl., 308 (2005), 290-302], [D. Q. Lu, H. M. Srivastava, Comput. Math. Appl., 62 (2011), 3591-3602] and [W. Wang, C. Jia, T. Wang, Comput. Math. Appl., 55 (2008), 1322-1332]). In this paper, we introduce and investigate an analogous generalization of Fubini polynomials of higher order, which we call bivariate ApostolFubini polynomials of higher order. We then obtain an explicit formula of these generalized Fubini polynomials and establish several symmetry identities. Moreover, we also establish relations of these polynomials with other Apostol-type numbers and polynomials.

Keywords: Fubini numbers, Fubini polynomials, ordered Bell numbers, geometric polynomials, Apostol-Fubini polynomials, generalized Fubini polynomials of higher order, Apostol-Bernoulli polynomials, Apostol-Euler polynomials, Apostol-Genocchi polynomials, Apostol-type polynomials, Stirling numbers of the second kind, Apostol-Stirling numbers, $\lambda$-Stirling numbers of the second kind, Gauss hypergeometric functions.
\end{abstract}

2020 MSC: 11B68, 11B73, 05A10, 11B65, 33C05, 11B83.

(C)2021 All rights reserved.

\section{Introduction}

Throughout this paper, we use the usual notations $\mathbb{N}, \mathbb{Z}, \mathbb{R}$, and $\mathbb{C}$ for the sets of natural numbers, integers, real numbers, and complex numbers respectively. Also, we let $\mathbb{N}_{0}:=\mathbb{N} \cup\{0\}, \mathbb{Z}^{-}:=\{-1,-2,-3, \ldots\}$, and $\mathbb{Z}_{0}^{-}:=\mathbb{Z}^{-} \cup\{0\}$.

The classical Fubini polynomials or geometric polynomials $F_{n}(y)$ are defined in [25] by

$$
F_{n}(y)=\sum_{k=0}^{n} S_{2}(n, k) k ! y^{k},
$$

where $S_{2}(n, k)$ is the Stirling numbers of the second kind [7]. These polynomials satisfy the generating function

$$
\frac{1}{1-y\left(e^{t}-1\right)}=\sum_{n=0}^{\infty} F_{n}(y) \frac{t^{n}}{n !},
$$

Email address: nestor .acala@gmail. com (Nestor G. Acala)

doi: $10.22436 /$ jmcs.023.01.02

Received: 2020-07-29 Revised: 2020-08-12 Accepted: 2020-08-17 
and the recurrence relation

$$
F_{n+1}(y)=y \frac{d}{d y}\left[F_{n}(y)+y F_{n}(y)\right] \quad(\text { see }[5])
$$

Setting $y=1$ in (1.1), we obtain the $n^{\text {th }}$ Fubini number or the ordered Bell number $F_{n}$, defined by

$$
F_{n}(1)=F_{n}=\sum_{k=0}^{n} S_{2}(n, k) k !
$$

Combinatorially, the number $F_{n}$ counts all the possible set partitions of an n-element set such that the order of the blocks matters.

As generalization of the classical Fubini polynomials, the bivariate Fubini polynomials of order $\alpha$ are defined through the generating function

$$
\frac{e^{x t}}{\left[1-y\left(e^{t}-1\right)\right]^{\alpha}}=\sum_{n=0}^{\infty} F_{n}^{(\alpha)}(x, y) \frac{t^{n}}{n !}(\text { see }[12,14-16]) .
$$

When $\alpha=1, F_{n}^{(\alpha)}(x, y):=F_{n}(x, y)$, the two-variable Fubini polynomials are given by

$$
\frac{e^{x t}}{1-y\left(e^{t}-1\right)}=\sum_{n=0}^{\infty} F_{n}(x, y) \frac{t^{n}}{n !}(\text { see }[9,10,13]) .
$$

Moreover, setting $x=0$ in (1.2), we obtain

$$
F_{n}^{(\alpha)}(0, y):=F_{n}^{(\alpha)}(y) \text { and } F_{n}^{(\alpha)}(1):=F_{n}^{(\alpha)},
$$

where $F_{n}^{(\alpha)}(y)$ and $F_{n}^{(\alpha)}$ are called the higher order Fubini polynomials and the higher order Fubini numbers respectively (see $[8,11]$ ).

Recently, a q-analogue of $F_{n}(y)$ called q-Fubini polynomials was introduced by Duran et al. [6] through the generating function

$$
\frac{1}{1-y\left(e_{q}(t)-1\right)}=\sum_{n=0}^{\infty} F_{n, q}(y) \frac{t^{n}}{[n]_{q} !},|q|<1,
$$

where

$$
[n]_{\mathrm{q}}:=\frac{\mathrm{q}^{\mathrm{n}}-1}{\mathrm{q}-1} \text { and } \mathrm{e}_{\mathrm{q}}(\mathrm{t}):=\sum_{n=0}^{\infty} \frac{\mathrm{t}^{\mathrm{n}}}{[\mathrm{n}]_{\mathrm{q}} !}
$$

are the $q$-analogues of the nonnegative integer $n$ and the exponential function $e^{t}$, respectively. When $y=1$ in (1.3), $F_{n, q}(1):=F_{n, q}$, the $n^{\text {th }} q$-Fubini numbers. Of course, as $q \rightarrow 1^{-}$, one can obtain

$$
\lim _{q \rightarrow 1^{-}} F_{n, q}(y)=F_{n}(y) .
$$

The classical Bernoulli polynomials $B_{n}(x)$, Euler polynomials $E_{n}(x)$, and Genocchi polynomials $G_{n}(x)$ together with their natural higher order generalizations $B_{n}^{(\alpha}(x), E_{n}^{(\alpha}(x)$, and $G_{n}^{(\alpha}(x)$ are usually defined by means of the generating functions (see [2-4])

$$
\left(\frac{t}{e^{t}-1}\right)^{\alpha} e^{x t}=\sum_{n=0}^{\infty} B_{n}^{(\alpha)}(x) \frac{t^{n}}{n !} \quad(|t|<2 \pi, \alpha \in \mathbb{C}),
$$




$$
\begin{aligned}
& \left(\frac{2}{e^{t}+1}\right)^{\alpha} e^{x t}=\sum_{n=0}^{\infty} E_{n}^{(\alpha)}(x) \frac{t^{n}}{n !} \quad(|t|<\pi, \alpha \in \mathbb{C}), \\
& \left(\frac{2 t}{e^{t}+1}\right)^{\alpha} e^{x t}=\sum_{n=0}^{\infty} G_{n}^{(\alpha)}(x) \frac{t^{n}}{n !} \quad(|t|<\pi, \alpha \in \mathbb{C}) .
\end{aligned}
$$

Hence,

$$
B_{n}^{(1)}(x):=B_{n}(x), \quad E_{n}^{(1)}(x):=E_{n}(x), \quad \text { and } \quad G_{n}^{(1)}(x):=G_{n}(x) .
$$

The classical Bernoulli numbers $B_{n}$, Euler numbers $E_{n}$, and Genocchi numbers $G_{n}$ are obtained by setting further $x=0$. That is

$$
B_{n}(0):=B_{n}, \quad E_{n}(0):=E_{n}, \quad \text { and } \quad G_{n}(0):=G_{n} .
$$

The higher order Apostol-Bernoulli polynomials $B_{n}^{(\alpha)}(x ; \lambda)$, higher order Apostol-Euler polynomials $E_{n}^{(\alpha)}(x ; \lambda)$, and higher order Apostol-Genocchi polynomials $G_{n}^{(\alpha)}(x ; \lambda)$ (see [17-21, 24, 27]) are defined through the generating functions:

$$
\begin{aligned}
& \left(\frac{t}{\lambda e^{t}-1}\right)^{(\alpha)} e^{x t}=\sum_{n=0}^{\infty} B_{n}^{(\alpha)}(x ; \lambda) \frac{t^{n}}{n !} \quad\left(|t|<2 \pi \text { if } \lambda=1,|t|<|\ln \lambda| \text { if } \lambda \neq 1 ; 1^{\alpha}=1, \alpha \in \mathbb{C}\right), \\
& \left(\frac{2}{\lambda e^{t}+1}\right)^{(\alpha)} e^{x t}=\sum_{n=0}^{\infty} E_{n}^{(\alpha)}(x ; \lambda) \frac{t^{n}}{n !} \quad\left(|t|<|\ln (-\lambda)| ; 1^{\alpha}=1, \alpha \in \mathbb{C}\right), \\
& \left(\frac{2 t}{\lambda e^{t}+1}\right)^{(\alpha)} e^{x t}=\sum_{n=0}^{\infty} G_{n}^{(\alpha)}(x ; \lambda) \frac{t^{n}}{n !} \quad\left(|t|<|\ln (-\lambda)| ; 1^{\alpha}=1, \alpha \in \mathbb{C}\right) .
\end{aligned}
$$

For $\lambda=1$,

$$
B_{n}^{(\alpha)}(x ; 1)=B_{n}^{\alpha}(x), \quad E_{n}^{(\alpha)}(x, 1)=E_{n}^{(\alpha)}(x), \quad \text { and } ; G_{n}^{(\alpha)}(x, 1)=G_{n}^{(\alpha)}(x),
$$

where $B_{n}^{\alpha}(x), E_{n}^{\alpha}(x)$, ang $G_{n}^{\alpha}(x)$ are the Bernoulli, Euler and Genocchi polynomials of order $\alpha$, respectively. Further setting $\alpha=1$, each reduces to its classical kind.

It follows directly from the generating functions (1.4), (1.5), and (1.6) (see also [26]) that

$$
\begin{aligned}
\lambda B_{n}^{(\alpha)}(x+1 ; \lambda)-B_{n}^{(\alpha)}(x ; \lambda) & =n B_{n-1}^{(\alpha-1)}(x ; \lambda), \\
\lambda E_{n}^{(\alpha)}(x+1 ; \lambda)+E_{n}^{(\alpha)}(x ; \lambda) & =2 E_{n}^{(\alpha-1)}(x ; \lambda), \\
\lambda G_{n}^{(\alpha)}(x+1 ; \lambda)+G_{n}^{(\alpha)}(x ; \lambda) & =2 n G_{n-1}^{(\alpha-1)}(x ; \lambda) .
\end{aligned}
$$

\section{Generalized Fubini-type polynomials}

Motivated by the definitions of the higher order Apostol-type polynomials mentioned above, we consider an analogous definition of higher order Apostol-Fubini polynomials and explore some of its properties.

Definition 2.1. Let $\alpha$ be an arbitrary real or complex number. We define the higher order bivariate Apostol-Fubini polynomials of order $\alpha$ through the generating function (see also [1, p.590])

$$
\begin{aligned}
& \frac{1}{\left(1-y\left(\lambda e^{t}-1\right)\right)^{\alpha}} e^{x t}=\sum_{n=0}^{\infty} F_{n}^{(\alpha)}(x, y ; \lambda) \frac{t^{n}}{n !}, \\
& \left(|t|<2 \pi \text { if } \lambda=\frac{y+1}{y},|t|<\left|\ln \left(\frac{y+1}{\lambda y}\right)\right| \text { if } \lambda \neq \frac{y+1}{y} ; 1^{\alpha}:=1 ; \lambda \in \mathbb{C}\right) .
\end{aligned}
$$


When $x=0$, we obtain the higher order univariate Apostol-Fubini polynomials $F_{n}^{(\alpha)}(y ; \lambda)$ given by

$$
\frac{1}{\left(1-y\left(\lambda e^{t}-1\right)\right)^{\alpha}}=\sum_{n=0}^{\infty} F_{n}^{(\alpha)}(y ; \lambda) \frac{t^{n}}{n !} .
$$

Taking $\lambda=1$ in (2.1), we obtain the higher order bivariate Fubini polynomials $F_{n}^{(\alpha)}(x, y)$.

Remark 2.2. Following the works of Srivastava [23] (see also Luo and Srivastava [21], Dere et al. [3]) on Apostol-type polynomials, the constraints on $|t|$ in Definition 2.1 are meant to ensure that the generating function (2.1) are analytic throughout the prescribed open disks in the complex t-plane (centred at the origin $t=0$ ) in order to have the corresponding convergent Taylor-Maclaurin series expansions (about the origin $t=0$ ) occurring on the right-hand side of (2.1) (with a positive radius of convergence). Moreover, throughout this investigation, $\ln z$ is tacitly assumed to denote the principal branch of the multi-valued function $\ln z$ of complex variable $z$ with the imaginary part $\mathfrak{I}(\ln z)$ constrained by $-\pi<\mathfrak{I}(\ln z) \leqslant \pi$. More importantly, throughout this presentation, wherever $\left|\ln \left(\frac{y+1}{\lambda y}\right)\right|$ and $\left|\ln \left(-\frac{y+1}{\lambda y}\right)\right|$ appear as the radii of the open disks in the complex $t$-plane (centred at the origin $t=0$ ) in which the defining generating functions are analytic, it is tacitly assumed that the obviously exceptional cases when $\frac{y+1}{\lambda y}=1$ and $\frac{y+1}{\lambda y}=-1$, respectively, are to be treated separately.

Theorem 2.3. The higher order bivariate Apostol-Fubini polynomials satisfy the following relations:

$$
\begin{aligned}
\lambda y F_{n}^{(\alpha)}(x+1, y ; \lambda) & =(y+1) F_{n}^{(\alpha)}(x, y ; \lambda)-F_{n}^{(\alpha-1)}(x, y ; \lambda), \\
\alpha \lambda y F_{n}^{(\alpha+1)}(x+1, y ; \lambda) & =F_{n+1}^{(\alpha)}(x, y ; \lambda)-x F_{n}^{(\alpha)}(x, y ; \lambda) .
\end{aligned}
$$

Proof. Applying (2.1), we have

$$
\begin{aligned}
\sum_{n=0}^{\infty} \lambda y F_{n}^{(\alpha)}(x+1, y ; \lambda) \frac{t^{n}}{n !} & =\frac{\lambda y e^{(x+1) t}}{\left(1-y\left(\lambda e^{t}-1\right)\right)^{\alpha}} \\
& =\frac{e^{x t}\left[(y+1)-\left(1-y\left(\lambda e^{t}-1\right)\right)\right]}{\left(1-y\left(\lambda e^{t}-1\right)\right)^{\alpha}} \\
& =\sum_{n=0}^{\infty}\left[(y+1) F_{n}^{(\alpha)}(x, y ; \lambda)-F_{n}^{(\alpha-1)}(x, y ; \lambda)\right] \frac{t^{n}}{n !}
\end{aligned}
$$

Comparing the coefficients of $\frac{\mathrm{t}^{\mathrm{n}}}{\mathrm{n} !}$ in (2.4) and (2.5), we get (2.2).

Now, differentiating both sides of (2.1) with respect to $t$, we get

$$
\frac{\alpha \lambda y}{\left(1-y\left(\lambda e^{t}-1\right)\right)^{\alpha+1}} e^{(x+1) t}+\frac{x}{\left(1-y\left(\lambda e^{t}-1\right)\right)^{\alpha}} e^{x t}=\sum_{n=0}^{\infty} F_{n+1}^{(\alpha)}(x, y ; \lambda) \frac{t^{n}}{n !} .
$$

Hence,

$$
\sum_{n=0}^{\infty}\left[\alpha \lambda y F_{n}^{(\alpha+1)}(x+1, y ; \lambda)+x F_{n}^{(\alpha)}(x, y ; \lambda)\right] \frac{t^{n}}{n !}=\sum_{n=0}^{\infty} F_{n+1}^{(\alpha)}(x, y ; \lambda) \frac{t^{n}}{n !} .
$$

Comparing the coefficients of $\frac{t^{n}}{n !}$, we get (2.3).

Theorem 2.4. For arbitrary real or complex number $\alpha$, we have

$$
\begin{aligned}
F_{n}^{(\alpha)}(x+z, y ; \lambda) & =\sum_{k=0}^{n}\left(\begin{array}{l}
n \\
k
\end{array}\right) F_{k}^{(\alpha)}(x, y ; \lambda) z^{n-k}, \quad z \neq 0, \\
& =\sum_{k=0}^{n}\left(\begin{array}{l}
n \\
k
\end{array}\right) F_{k}^{(\alpha)}(z, y ; \lambda) x^{n-k}, \quad x \neq 0 .
\end{aligned}
$$


Proof. Note that

$$
\begin{aligned}
\sum_{n=0}^{\infty} F_{n}^{(\alpha)}(x+z, y ; \lambda) \frac{t^{n}}{n !} & =\frac{1}{\left(1-y\left(\lambda e^{t}-1\right)\right)^{\alpha}} e^{(x+z) t} \\
& =\frac{1}{\left(1-y\left(\lambda e^{t}-1\right)\right)^{\alpha}} e^{x t} \cdot e^{z t} \\
& =\sum_{n=0}^{\infty} F_{n}^{(\alpha)}(x, y ; \lambda) \frac{t^{n}}{n !} \sum_{n=0}^{\infty} z^{n} \frac{t^{n}}{n !} \\
& =\sum_{n=0}^{\infty} \sum_{k=0}^{n}\left(\begin{array}{l}
n \\
k
\end{array}\right) F_{k}^{(\alpha)}(x, y ; \lambda) z^{n-k} \frac{t^{n}}{n !}
\end{aligned}
$$

Comparing the coefficients of $\frac{\mathrm{t}^{\mathrm{n}}}{\mathrm{n} !}$, we obtain (2.6); and interchanging the roles of $x$ and $z$, we obtain (2.7).

Taking $z=0$ in (2.7), we obtain a relationship between the bivariate Apostol-Fubini polynomials $F_{n}^{(\alpha)}(x, y ; \lambda)$ and univariate Apostol-Fubini polynomials $F_{n}^{(\alpha)}(y ; \lambda)$ in the next corollary.

Corollary 2.5. For $\mathrm{n} \in \mathbb{N}_{0}$ and $x \neq 0$,

$$
F_{n}^{(\alpha)}(x, y ; \lambda)=\sum_{k=0}^{n}\left(\begin{array}{l}
n \\
k
\end{array}\right) F_{k}^{(\alpha)}(y ; \lambda) x^{n-k}
$$

For the basic derivative and integral properties of $F_{n}^{(\alpha)}(x, y ; a, b, c)$, we have the following theorem.

Theorem 2.6. For arbitrary real or complex number $\alpha$,

$$
\begin{aligned}
\frac{d}{d x} F_{n}^{(\alpha)}(x, y ; \lambda) & =n \cdot F_{n-1}^{(\alpha)}(x, y ; \lambda), \\
\int F_{n}^{(\alpha)}(x, y ; \lambda) d x & =\frac{1}{(n+1)} F_{n+1}^{(\alpha)}(x, y ; \lambda) .
\end{aligned}
$$

Proof. It follows from Corollary 2.5 that

$$
\frac{d}{d x} F_{n}^{(\alpha)}(x, y ; \lambda)=\sum_{k=0}^{n-1}\left(\begin{array}{l}
n \\
k
\end{array}\right)(n-k) F_{k}^{(\alpha)}(y ; \lambda) x^{n-k-1}=n \sum_{k=0}^{n-1}\left(\begin{array}{c}
n-1 \\
k
\end{array}\right) F_{k}^{(\alpha)}(y ; \lambda) x^{(n-1)-k}=n F_{n-1}^{(\alpha)}(x, y ; \lambda) .
$$

Equation (2.9) follows directly from (2.8).

Note that for any real number $\alpha$,

$$
\left(\frac{e^{x t}}{1-y\left(\lambda e^{t}-1\right)}\right)^{\alpha}=\sum_{n=0}^{\infty} F_{n}^{(\alpha)}(\alpha x, y ; \lambda) \frac{t^{n}}{n !} .
$$

Separating the right-hand side of (2.10) in terms of the parity of the indices using the identity

$$
\sum_{n=0}^{\infty} f_{n}(w)=\sum_{n=0}^{\infty} f_{2 n}(w)+\sum_{n=0}^{\infty} f_{2 n+1}(w)
$$

and then replacing $t$ by it, where $i^{2}=-1$, we obtain the next identities involving trigonometric functions and Apostol-Fubini polynomials. 
Theorem 2.7. The following summation formulas for higher order bivariate Apostol-Fubini polynomials hold true:

$$
\begin{aligned}
& {\left[\frac{(y+1) \cos (x t)-\lambda y \cos (t-x t)}{N(y, \lambda, t)}\right]^{\alpha}=\sum_{n=0}^{\infty} F_{2 n}^{(\alpha)}(\alpha x, y ; \lambda) \frac{(-1)^{n} t^{2 n}}{(2 n) !}} \\
& {\left[\frac{(y+1) \sin (x t)-\lambda y \sin (t-x t)}{N(y, \lambda, t)}\right]^{\alpha}=\sum_{n=0}^{\infty} F_{2 n+1}^{(\alpha)}(\alpha x, y ; \lambda) \frac{(-1)^{n} t^{2 n+1}}{(2 n+1) !}}
\end{aligned}
$$

where $N(y, \lambda, t)=(y+1)^{2}+(\lambda y)^{2}-2 \lambda y(y+1) \cos t$

Setting $\alpha=\lambda=y=1$, and $x=0$ in Theorem 2.7, we obtain the well-known formula involving the classical Fubini numbers.

Corollary 2.8. The following summation formulas for Fubini numbers hold true:

$$
\frac{2-\cos t}{5-4 \cos t}=\sum_{n=0}^{\infty} F_{2 n} \frac{(-1)^{n} t^{2 n}}{(2 n) !}, \quad \frac{\sin t}{5-4 \cos t}=\sum_{n=0}^{\infty} F_{2 n+1} \frac{(-1)^{n} t^{2 n+1}}{(2 n+1) !}
$$

\section{Explicit formulas of $F_{\mathfrak{n}}^{(\alpha)}(x, y ; \lambda)$}

In this section, we obtain some series representations of the polynomials $F_{n}^{(\alpha)}(x, y ; \lambda)$.

First, we give the representation of higher order bivariate Apostol-Fubini polynomials $F_{n}^{(\alpha)}(x, y ; \lambda)$ in terms of the higher order bivariate Fubini polynomials $F_{n}^{(\alpha)}(x, y)$ in the next theorem.

Theorem 3.1. For $\alpha, \lambda \in \mathbb{C}$ and $n \in \mathbb{N}_{0}$,

$$
F_{n}^{(\alpha)}(x, y ; \lambda)=\lambda^{-x} \sum_{k=0}^{\infty} F_{n+k}^{(\alpha)}(x, y) \frac{(\ln \lambda)^{k}}{k !} .
$$

Proof. With the aid of (2.1) and (1.2), we have

$$
\begin{aligned}
\sum_{n=0}^{\infty} F_{n}^{(\alpha)}(x, y ; \lambda) \frac{t^{n}}{n !} & =e^{-x \ln \lambda} \cdot \frac{e^{x(t+\ln \lambda)}}{\left(1-y\left(e^{t+\ln \lambda}-1\right)\right)^{\alpha}} \\
& =e^{-x \ln \lambda} \sum_{k=0}^{\infty} F_{k}^{(\alpha)}(x, y) \frac{(t+\ln \lambda)^{k}}{k !} \\
& =e^{-x \ln \lambda} \sum_{k=0}^{\infty} F_{k}^{(\alpha)}(x, y) \sum_{n=0}^{k} \frac{t^{n}(\ln \lambda)^{k-n}}{(k-n) ! n !} \\
& =e^{-x \ln \lambda} \sum_{n=0}^{\infty}\left[\sum_{k=0}^{\infty} F_{n+k}^{(\alpha)}(x, y) \frac{(\ln \lambda)^{k}}{k !}\right] \frac{t^{n}}{n !}
\end{aligned}
$$

Thus, comparing the coefficients of $\frac{t^{n}}{n !}$, we obtain desired result.

Now, we establish an explicit expression of $F_{n}^{(r)}(x, y ; \lambda)$ in terms of the Gaussian hypergeometric function ${ }_{2} \mathrm{~F}_{1}(\mathrm{a}, \mathrm{b} ; \mathrm{c} ; z)$ which is given by

$$
{ }_{2} \mathrm{~F}_{1}(\mathrm{a}, \mathrm{b} ; \mathrm{c} ; z):=\sum_{\mathrm{n}=0}^{\infty} \frac{(\mathrm{a})_{\mathrm{n}}(\mathrm{b})_{\mathrm{n}}}{(\mathrm{c})_{\mathrm{n}}} \frac{z^{\mathrm{n}}}{\mathrm{n} !}
$$

where $c \notin \mathbb{Z}_{0} ;|z|<1 ; z=1$ and $\mathfrak{R}(\mathrm{c}-\mathrm{a}-\mathrm{b})>0 ; z=-1$ and $\mathfrak{R}(\mathrm{c}-\mathrm{a}-\mathrm{b})>-1$. Here, $(\mathrm{q})_{0}=1$, and $(q)_{n}=q(q+1) \cdots(q+n-1)$ for $n>0$. 
Theorem 3.2. For $n, r \in \mathbb{N}_{0}$, we have

$$
F_{n}^{(r)}(x, y ; \lambda)=\sum_{i=0}^{n}\left(\begin{array}{c}
n \\
i
\end{array}\right)\left(\begin{array}{c}
r+i-1 \\
i
\end{array}\right) \sum_{m=0}^{i}(-1)^{m} \lambda^{m} m^{i}\left(\begin{array}{c}
i \\
m
\end{array}\right)(x+m)^{n-i}{ }_{2} F_{1}\left(-n+i, i ; 1+i ; \frac{m}{m+x}\right) .
$$

Proof. By (2.1), we have

$$
F_{n}^{(r)}(x, y ; \lambda)=D_{t}^{n}\left[\left(1-y\left(\lambda e^{t}-1\right)\right)^{-r} e^{x t}\right]_{t=0}
$$

where $D_{t}:=\frac{d}{d t}$. Thus,

$$
F_{n}^{(r)}(x, y ; \lambda)=\sum_{s=0}^{n}\left(\begin{array}{l}
n \\
s
\end{array}\right) x^{n-s} D_{t}^{s}\left[\left(1-y\left(\lambda e^{t}-1\right)\right)^{-r}\right]_{t=0}
$$

Using

$$
(A+w)^{-r}=\sum_{i=0}^{\infty}\left(\begin{array}{c}
r+i-1 \\
i
\end{array}\right) A^{-r-i}(-w)^{i}, \quad(|w|<|A|)
$$

and

$$
\left(\lambda e^{t}-1\right)^{i}=i ! \sum_{j=1}^{\infty} S(j, i ; \lambda) \frac{t^{j}}{j !},
$$

where $S(j, i ; \lambda)$ is the $\lambda$-Stirling numbers of the second kind (see [21]) we obtain

$$
F_{n}^{(r)}(x, y ; \lambda)=\sum_{s=0}^{n}\left(\begin{array}{l}
n \\
s
\end{array}\right) x^{n-s} \sum_{i=0}^{s}\left(\begin{array}{c}
r+i-1 \\
i
\end{array}\right) y^{i} i ! S(s, i ; \lambda)
$$

Using the explicit formula

$$
S(j, i ; \lambda)=\frac{1}{i !} \sum_{m=0}^{i}(-1)^{i-m}\left(\begin{array}{c}
i \\
m
\end{array}\right) \lambda^{m} m^{j}
$$

and the identity

$$
\left(\begin{array}{l}
n \\
s
\end{array}\right)=\left(\begin{array}{c}
n \\
n-s
\end{array}\right)
$$

we get

$$
\begin{aligned}
F_{n}^{(r)}(x, y ; \lambda) & =\sum_{s=0}^{n}\left(\begin{array}{c}
n \\
s
\end{array}\right) \sum_{i=0}^{s}\left(\begin{array}{c}
r+i-1 \\
i
\end{array}\right) y^{i} x^{n-s} \sum_{m=0}^{i}(-1)^{i-m}\left(\begin{array}{c}
i \\
m
\end{array}\right) \lambda^{m} m^{s} \\
& =\sum_{i=0}^{n} \sum_{s=i}^{n}\left(\begin{array}{c}
n \\
n-s
\end{array}\right)\left(\begin{array}{c}
r+i-1 \\
i
\end{array}\right)(-y)^{i} x^{n-s} \sum_{m=0}^{i}(-\lambda)^{m}\left(\begin{array}{c}
i \\
m
\end{array}\right) m^{s} \\
& =\sum_{i=0}^{n} \sum_{s=0}^{n-i}\left(\begin{array}{c}
n \\
n-s-i
\end{array}\right)\left(\begin{array}{c}
r+i-1 \\
i
\end{array}\right)(-y)^{i} x^{n-s-i} \sum_{m=0}^{i}(-\lambda)^{m}\left(\begin{array}{c}
i \\
m
\end{array}\right) m^{s+i}
\end{aligned}
$$

Using the identity

$$
(n-s-i) !=\frac{(-1)^{s}(n-i) !}{(-n+i)_{s}}
$$

gives

$$
F_{n}^{(r)}(x, y ; \lambda)=\sum_{i=0}^{n}\left(\begin{array}{c}
n \\
i
\end{array}\right)\left(\begin{array}{c}
r+i-1 \\
i
\end{array}\right)(-y)^{i} \sum_{m=0}^{i}(-\lambda)^{m}\left(\begin{array}{c}
i \\
m
\end{array}\right) m^{i} x^{n-i}{ }_{2} F_{1}\left(-n+i, 1 ; 1+i ; \frac{-m}{x}\right)
$$


Finally, applying Pfaff-Kummer hypergeometric transformation

$$
{ }_{2} \mathrm{~F}_{1}(\mathrm{a}, \mathrm{b} ; \mathrm{c} ; z)=(1-z)^{-\mathrm{a}}{ }_{2} \mathrm{~F}_{1}\left(\mathrm{a}, \mathrm{c}-\mathrm{b} ; \mathrm{c} ; \frac{z}{z-1}\right) \quad\left(\mathrm{c} \notin \mathbb{Z}_{0}^{-} ;|\arg (1-z)| \leqslant \pi-\epsilon(0<\epsilon<\pi)\right),
$$

yields

$$
F_{n}^{(r)}(x, y ; \lambda)=\sum_{i=0}^{n}\left(\begin{array}{c}
n \\
i
\end{array}\right)\left(\begin{array}{c}
r+i-1 \\
i
\end{array}\right)(-y)^{i} \sum_{m=0}^{i}(-\lambda)^{m}\left(\begin{array}{c}
i \\
m
\end{array}\right) m^{i}(x+m)^{n-i}{ }_{2} F_{1}\left(-n+i, i ; 1+i ; \frac{m}{m+x}\right) .
$$

Remark 3.3. It follows from the proof of Theorem 3.2 that $F_{n}^{(\alpha)}(x, y ; \lambda)$ can be written explicitly in terms of the Apostol-Stirling numbers of the second kind:

$$
F_{n}^{(r)}(x, y ; \lambda)=\sum_{s=0}^{n}\left(\begin{array}{l}
n \\
s
\end{array}\right) x^{n-s} \sum_{i=0}^{s}\left(\begin{array}{c}
r+i-1 \\
i
\end{array}\right) y^{i} i ! S(s, i ; \lambda)
$$

\section{Symmetry identities for $F_{n}^{(\alpha)}(x, y ; \lambda)$}

We now derive some symmetry identities for the higher order bivariate Apostol-Fubini polynomials $F_{n}^{(\alpha)}(x, y ; \lambda)$.

Theorem 4.1. For integers $\mathrm{a}, \mathrm{b}$ and $\mathrm{n} \in \mathbb{N}_{0}$, we have

$$
\sum_{k=0}^{n}\left(\begin{array}{l}
n \\
k
\end{array}\right) a^{k} b^{n-k} F_{k}^{(\alpha)}(b x, y ; \lambda) F_{n-k}^{(\alpha)}(a z, y ; \lambda)=\sum_{k=0}^{n}\left(\begin{array}{l}
n \\
k
\end{array}\right) b^{k} a^{n-k} F_{k}^{(\alpha)}(a x, y ; \lambda) F_{n-k}^{(\alpha)}(b z, y ; \lambda) .
$$

Proof. Consider

$$
A(t)=\frac{e^{a b(x+z) t}}{\left(1-y\left(\lambda e^{a t}-1\right)\right)^{\alpha}\left(1-y\left(\lambda e^{b t}-1\right)\right)^{\alpha}} .
$$

Expanding $A(t)$ into a series, we obtain

$$
\begin{aligned}
A(t) & =\frac{e^{b x(a t)}}{\left(1-y\left(\lambda e^{a t}-1\right)^{\alpha}\right.} \cdot \frac{e^{a z(b t)}}{\left(1-y\left(\lambda e^{b t}-1\right)^{\alpha}\right.} \\
& =\sum_{n=0}^{\infty} F_{n}^{(\alpha)}(b x, y ; \lambda) \frac{(a t)^{n}}{n !} \cdot \sum_{n=0}^{\infty} F_{n}^{(\alpha)}(a z, y ; \lambda) \frac{(b t)^{n}}{n !} \\
& =\sum_{n=0}^{\infty} \sum_{k=0}^{n}\left(\begin{array}{l}
n \\
k
\end{array}\right) a^{k} b^{n-k} F_{k}^{(\alpha)}(b x, y ; \lambda) F_{n-k}^{(\alpha)}(a z, y ; \lambda) \frac{t^{n}}{n !}
\end{aligned}
$$

Similarly,

$$
\begin{aligned}
A(t) & =\frac{e^{a x(b t)}}{\left(1-y\left(\lambda e^{b t}-1\right)\right)^{\alpha}} \cdot \frac{e^{b z(a t)}}{\left(1-y\left(\lambda e^{a t}-1\right)\right)^{\alpha}} \\
& =\sum_{n=0}^{\infty} F_{n}^{(\alpha)}(a x, y ; \lambda) \frac{(b t)^{n}}{n !} \cdot \sum_{n=0}^{\infty} F_{n}^{(\alpha)}(b z, y ; \lambda) \frac{(a t)^{n}}{n !} \\
& =\sum_{n=0}^{\infty} \sum_{k=0}^{n}\left(\begin{array}{l}
n \\
k
\end{array}\right) b^{k} a^{n-k} F_{k}^{(\alpha)}(a x, y ; \lambda) F_{n-k}^{(\alpha)}(b z, y ; \lambda) \frac{t^{n}}{n !} .
\end{aligned}
$$

Comparing (4.1) and (4.2), we get the desired identity. 
Theorem 4.2. For positive integers $\mathrm{a}, \mathrm{b}$ and $\mathrm{n} \in \mathbb{N}_{0}$, we have

$$
\begin{aligned}
\sum_{k=0}^{n}\left(\begin{array}{l}
n \\
k
\end{array}\right) \sum_{i=0}^{a-1} \sum_{j=0}^{b-1} a^{k} b^{n-k} F_{k}^{(\alpha)}\left(b x+\frac{b}{a} i, y ; \lambda\right) F_{n-k}^{(\alpha)}\left(a z+\frac{a}{b} j, y ; \lambda\right) \\
=\sum_{k=0}^{n}\left(\begin{array}{l}
n \\
k
\end{array}\right) \sum_{i=0}^{b-1} \sum_{j=0}^{a-1} b^{k} a^{n-k} F_{k}^{(\alpha)}\left(a x+\frac{a}{b} i, y ; \lambda\right) F_{n-k}^{(\alpha)}\left(b z+\frac{b}{a} j, y ; \lambda\right) .
\end{aligned}
$$

Proof. Consider

$$
B(t)=\frac{e^{a b(x+z) t}\left(e^{a b t}-1\right)^{2}}{\left(1-y\left(\lambda e^{a t}-1\right)\right)^{\alpha}\left(1-y\left(\lambda e^{b t}-1\right)\right)^{\alpha}}\left(e^{a t}-1\right)\left(e^{b t}-1\right) .
$$

Expanding $B(t)$ into a series, we get

$$
\begin{aligned}
B(t) & =\frac{e^{b x(a t)}}{\left(1-y\left(\lambda e^{a t}-1\right)\right)^{\alpha}} \frac{e^{a b t}-1}{e^{b t}-1} \cdot \frac{e^{a z(b t)}}{\left(1-y\left(\lambda e^{b t}-1\right)\right)^{\alpha}} \frac{e^{a b t}-1}{e^{a t}-1} \\
& =\frac{e^{b x(a t)}}{\left(1-y\left(\lambda e^{a t}-1\right)\right)^{\alpha}} \sum_{i=0}^{a-1} e^{b t i} \cdot \frac{e^{a z(b t)}}{\left(1-y\left(\lambda e^{b t}-1\right)\right)^{\alpha}} \sum_{j=0}^{b-1} e^{a t j} \\
& =\left(\sum_{n=0}^{\infty} \sum_{i=0}^{a-1} F_{n}^{(\alpha)}\left(b x+\frac{b}{a} i, y ; \lambda\right) \frac{(a t)^{n}}{n !}\right)\left(\sum_{n=0}^{\infty} \sum_{j=0}^{b-1} F_{n}^{(\alpha)}\left(a z+\frac{a}{b} j, y ; \lambda\right) \frac{(b t)^{n}}{n !}\right) \\
& =\sum_{n=0}^{\infty} \sum_{k=0}^{n}\left(\begin{array}{l}
n \\
k
\end{array}\right) \sum_{i=0}^{a-1} \sum_{j=0}^{b-1} a^{k} b^{n-k} F_{k}^{(\alpha)}\left(b x+\frac{b}{a} i, y ; \lambda\right) F_{n-k}^{(\alpha)}\left(a z+\frac{a}{b} j, y ; \lambda\right) \frac{t^{n}}{n !} .
\end{aligned}
$$

Similarly,

$$
\begin{aligned}
B(t) & =\frac{e^{a x(b t)}}{\left(1-y\left(\lambda e^{b t}-1\right)\right)^{\alpha}} \frac{e^{a b t}-1}{e^{a t}-1} \cdot \frac{e^{b z(a t)}}{\left(1-y\left(\lambda e^{a t}-1\right)\right)^{\alpha}} \frac{e^{a b t}-1}{e^{b t}-1} \\
& =\left(\sum_{n=0}^{\infty} \sum_{i=0}^{b-1} F_{n}^{(\alpha)}\left(a x+\frac{a}{b} i, y ; \lambda\right) \frac{(b t)^{n}}{n !}\right)\left(\sum_{n=0}^{\infty} \sum_{j=0}^{a-1} F_{n}^{(\alpha)}\left(b z+\frac{b}{a} j, y ; \lambda\right) \frac{(a t)^{n}}{n !}\right) \\
& =\sum_{n=0}^{\infty} \sum_{k=0}^{n}\left(\begin{array}{l}
n \\
k
\end{array}\right) \sum_{i=0}^{a-1} \sum_{j=0}^{b-1} b^{k} a^{n-k} F_{k}^{(\alpha)}\left(a x+\frac{a}{b} i, y ; \lambda\right) F_{n-k}^{(\alpha)}\left(b z+\frac{b}{a} j, y ; \lambda\right) \frac{t^{n}}{n !}
\end{aligned}
$$

Comparing (4.3) and (4.4) yields the desired result.

Theorem 4.3. For positive integers $\mathrm{a}, \mathrm{b}$ and $\mathrm{n} \in \mathbb{N}_{0}$, we have

$$
\begin{aligned}
\sum_{k=0}^{n}\left(\begin{array}{l}
n \\
k
\end{array}\right) \sum_{i=0}^{a-1} \sum_{j=0}^{b-1} a^{k} b^{n-k} F_{k}^{(\alpha)}\left(b x+\frac{b}{a} i+j, y ; \lambda\right) F_{n-k}^{(\alpha)}(a z, y ; \lambda) \\
=\sum_{k=0}^{n}\left(\begin{array}{l}
n \\
k
\end{array}\right) \sum_{i=0}^{b-1} \sum_{j=0}^{a-1} b^{k} a^{n-k} F_{k}^{(\alpha)}\left(a x+\frac{a}{b} i+j, y ; \lambda\right) F_{n-k}^{(\alpha)}(b z, y ; \lambda) .
\end{aligned}
$$

Proof. Consider

$$
C(t)=\frac{e^{a b(x+z) t}\left(e^{a b t}-1\right)^{2}}{\left(1-y\left(\lambda e^{a t}-1\right)\right)^{\alpha}\left(1-y\left(\lambda e^{b t}-1\right)\right)^{\alpha}}\left(e^{a t}-1\right)\left(e^{b t}-1\right) .
$$


Expanding $B(t)$ into a series, we get

$$
\begin{aligned}
C(t) & =\frac{e^{b x(a t)}}{\left(1-y\left(\lambda e^{a t}-1\right)\right)^{\alpha}} \frac{e^{a b t}-1}{e^{b t}-1} \cdot \frac{e^{a z(b t)}}{\left(1-y\left(\lambda e^{b t}-1\right)\right)^{\alpha}} \frac{e^{a b t}-1}{e^{a t}-1} \\
& =\frac{e^{b x(a t)}}{\left(1-y\left(\lambda e^{a t}-1\right)\right)^{\alpha}} \sum_{i=0}^{a-1} e^{b t i} \cdot \frac{e^{a z(b t)}}{\left(1-y\left(\lambda e^{b t}-1\right)\right)^{\alpha}} \sum_{j=0}^{b-1} e^{a t j} \\
& =\left(\sum_{n=0}^{\infty} \sum_{i=0}^{a-1} \sum_{j=0}^{b-1} F_{n}^{(\alpha)}\left(b x+\frac{b}{a} i+j, y ; \lambda\right) \frac{(a t)^{n}}{n !}\right) \sum_{n=0}^{\infty} F_{n}^{(\alpha)}(a z, y ; \lambda) \frac{(b t)^{n}}{n !} \\
& =\sum_{n=0}^{\infty} \sum_{k=0}^{n}\left(\begin{array}{l}
n \\
k
\end{array}\right) \sum_{i=0}^{a-1} \sum_{j=0}^{b-1} a^{k} b^{n-k} F_{k}^{(\alpha)}\left(b x+\frac{b}{a} i+j, y ; \lambda\right) F_{n-k}^{(\alpha)}(a z, y ; \lambda) \frac{t^{n}}{n !} .
\end{aligned}
$$

Similarly,

$$
\begin{aligned}
C(t) & =\frac{e^{a x(b t)}}{\left(1-y\left(\lambda e^{b t}-1\right)\right)^{\alpha}} \frac{e^{a b t}-1}{e^{a t}-1} \cdot \frac{e^{b z(a t)}}{\left(1-y\left(\lambda e^{a t}-1\right)\right)^{\alpha}} \frac{e^{a b t}-1}{e^{b t}-1} \\
& =\left(\sum_{n=0}^{\infty} \sum_{i=0}^{b-1} \sum_{j=0}^{a-1} F_{n}^{(\alpha)}\left(a x+\frac{a}{b} i+j, y ; \lambda\right) \frac{(b t)^{n}}{n !}\right) \sum_{n=0}^{\infty} F_{n}^{(\alpha)}(b z, y ; \lambda) \frac{(a t)^{n}}{n !} \\
& =\sum_{n=0}^{\infty} \sum_{k=0}^{n}\left(\begin{array}{l}
n \\
k
\end{array}\right) \sum_{i=0}^{b-1} \sum_{j=0}^{a-1} b^{k} a^{n-k} F_{k}^{(\alpha)}\left(a x+\frac{a}{b} i+j, y ; \lambda\right) F_{n-k}^{(\alpha)}(b z, y ; \lambda) \frac{t^{n}}{n !} .
\end{aligned}
$$

Comparing (4.5) and (4.6) yields the desired result.

In the next result, we establish symmetry identitity involving generalized Apostol-Fubini polynomials and the generalized sum of powers.

For each $k \in \mathbb{N}_{0}$, the sum of integer powers $S_{k}(n)$ is defined by $\mathcal{S}_{k}(n)=\sum_{j=0}^{n-1} j^{k}$ and has the exponential generating function

$$
\sum_{k=0}^{\infty} \mathcal{S}_{k}(n) \frac{t^{k}}{k !}=\frac{e^{n t}-1}{e^{t}-1} .
$$

In [22], Lu and Srivastava defined the generalized sum of integer powers $\mathcal{S}_{k}(n ; \lambda)$ through the generating function

$$
\sum_{k=0}^{\infty} \mathcal{S}_{k}(n ; \lambda) \frac{t^{k}}{k !}=\frac{\lambda e^{n t}-1}{\lambda e^{t}-1}(\lambda \in \mathbb{C})
$$

Clearly, $\mathcal{S}_{k}(n ; 1)=\mathcal{S}_{k}(n)$.

Theorem 4.4. For $\mathrm{a}, \mathrm{b} \in \mathbb{N} ; \mathrm{n} \in \mathbb{N}_{0}$; and $\mathrm{y} \neq-1$, we have

$$
\begin{aligned}
\sum_{k=0}^{n}\left(\begin{array}{l}
n \\
k
\end{array}\right) a^{n-k} b^{k} & F_{n-k}^{(\alpha)}(b x, y ; \lambda) \sum_{l=0}^{k}\left(\begin{array}{l}
k \\
l
\end{array}\right) \mathcal{S}_{l}\left(a ; \frac{\lambda y}{y+1}\right) F_{k-l}^{(\alpha-1)}(a z, y ; \lambda) \\
& =\sum_{k=0}^{n}\left(\begin{array}{l}
n \\
k
\end{array}\right) b^{n-k} a^{k} F_{n-k}^{(\alpha)}(a x, y ; \lambda) \sum_{l=0}^{k}\left(\begin{array}{l}
k \\
l
\end{array}\right) \mathcal{S}_{l}\left(b ; \frac{\lambda y}{y+1}\right) F_{k-l}^{(\alpha-1)}(b z, y ; \lambda) .
\end{aligned}
$$


Proof. Let

$$
D(t)=\frac{e^{a b(x+z) t}\left(1-y\left(\lambda e^{a b t}-1\right)\right)}{\left(1-y\left(\lambda e^{a t}-1\right)\right)^{\alpha}\left(1-y\left(\lambda e^{b t}-1\right)\right)^{\alpha}} .
$$

Grouping factors and expanding $\mathrm{D}(\mathrm{t})$ into series, we obtain

$$
\begin{aligned}
D(t) & =\frac{e^{b x(a t)}}{\left(1-y\left(\lambda e^{a t}-1\right)\right)^{\alpha}} \cdot \frac{1-y\left(\lambda e^{a b t}-1\right)}{1-y\left(\lambda e^{b t}-1\right)} \cdot \frac{e^{a z(b t)}}{1-y\left(\lambda e^{b t}-1\right)^{\alpha-1}} \\
& =\left(\sum_{n=0}^{\infty} F_{n}^{(\alpha)}(b x, y ; \lambda) \frac{(a t)^{n}}{n !}\right)\left(\sum_{n=0}^{\infty} \mathcal{S}_{n}\left(a, \frac{\lambda y}{y+1}\right) \frac{(b t)^{n}}{n !} \cdot \sum_{n=0}^{\infty} F_{n}^{(\alpha-1)}(a z, y ; \lambda) \frac{(b t)^{n}}{n !}\right) \\
& =\left(\sum_{n=0}^{\infty} F_{n}^{(\alpha)}(b x, y ; \lambda) \frac{(a t)^{n}}{n !}\right)\left(\sum_{n=0}^{\infty} \sum_{l=0}^{n}\left(\begin{array}{c}
n \\
l
\end{array}\right) b^{n} \mathcal{S}_{l}\left(a, \frac{\lambda y}{y+1}\right) F_{n-l}^{(\alpha-1)}(a z, y ; \lambda) \frac{t^{n}}{n !}\right) \\
& =\sum_{n=0}^{\infty}\left(\sum_{k=0}^{n}\left(\begin{array}{l}
n \\
k
\end{array}\right) a^{n-k} b^{k} F_{n-k}^{(\alpha)}(b x, y ; \lambda) \sum_{l=0}^{k}\left(\begin{array}{l}
k \\
l
\end{array}\right) \mathcal{S}_{l}\left(a, \frac{\lambda y}{y+1}\right) F_{k-l}^{(\alpha-1)}(a z, y ; \lambda)\right) \frac{t^{n}}{n !} .
\end{aligned}
$$

Similarly,

$$
\begin{aligned}
D(t) & =\frac{e^{a x(b t)}}{\left(1-y\left(\lambda e^{b t}-1\right)\right)^{\alpha}} \cdot \frac{1-y\left(\lambda e^{a b t}-1\right)}{1-y\left(\lambda e^{a t}-1\right)} \cdot \frac{e^{b z(a t)}}{1-y\left(\lambda e^{a t}-1\right)^{\alpha-1}} \\
& =\left(\sum_{n=0}^{\infty} F_{n}^{(\alpha)}(a x, y ; \lambda) \frac{(b t)^{n}}{n !}\right)\left(\sum_{n=0}^{\infty} \mathcal{S}_{n}\left(b, \frac{\lambda y}{y+1}\right) \frac{(a t)^{n}}{n !} \cdot \sum_{n=0}^{\infty} F_{n}^{(\alpha-1)}(b z, y ; \lambda) \frac{(a t)^{n}}{n !}\right) \\
& =\sum_{n=0}^{\infty}\left(\sum_{k=0}^{n}\left(\begin{array}{l}
n \\
k
\end{array}\right) b^{n-k} a^{k} F_{n-k}^{(\alpha)}(a x, y ; \lambda) \sum_{l=0}^{k}\left(\begin{array}{l}
k \\
l
\end{array}\right) \mathcal{S}_{l}\left(b, \frac{\lambda y}{y+1}\right) F_{k-l}^{(\alpha-1)}(b z, y ; \lambda)\right) \frac{t^{n}}{n !} .
\end{aligned}
$$

Comparing (4.7) and (4.8) yields the desired result.

\section{Some identities involving Apostol-type numbers and polynomials}

In this section, we establish some identities which relate higher order Apostol-Fubini polynomials to the Apostol-Stirling numbers of the second kind, higher order Apostol-Bernoulli polynomials, higher order Apostol-Euler polynomials, and higher order Apostol-Genocchi polynomials.

\subsection{Relations betweeen higher order Apostol-Fubini polynomials and $\lambda$-Stirling numbers of the second kind}

Theorem 5.1. For $\lambda \in \mathbb{C}$ and $\alpha, n \in \mathbb{N}_{0}$, we have

$$
F_{n}^{(\alpha)}(x, y ; \lambda)=\sum_{j=0}^{n}\left(\begin{array}{l}
n \\
j
\end{array}\right) \sum_{k=0}^{j}(\alpha+k-j){ }_{k} S_{2}(j, k ; \lambda) y^{k} x^{n-j}
$$

Proof. Note that

$$
\begin{aligned}
\left(1-y\left(\lambda e^{t}-1\right)\right)^{-\alpha} & =\sum_{k=0}^{\infty}\left(\begin{array}{c}
\alpha+k-1 \\
k
\end{array}\right) y^{k}\left(\lambda e^{t}-1\right)^{k} \\
& =\sum_{k=0}^{\infty}(\alpha+k-1)_{k} y^{k} \sum_{n=k}^{\infty} S_{2}(n, k ; \lambda) \frac{t^{n}}{n !}=\sum_{n=0}^{\infty} \sum_{k=0}^{n}(\alpha+k-1)_{k} y^{k} S_{2}(n, k ; \lambda) \frac{t^{n}}{n !} .
\end{aligned}
$$


Thus, using (2.1), we obtain

$$
\begin{aligned}
\sum_{n=0}^{\infty} F_{n}^{(\alpha)}(x, y, ; \lambda) \frac{t^{n}}{n !} & =\sum_{n=0}^{\infty} \sum_{k=0}^{n}(\alpha+k-1)_{k} y^{k} S_{2}(n, k ; \lambda) \frac{t^{n}}{n !} \cdot \sum_{n=0}^{\infty} x^{n} \frac{t^{n}}{n !} \\
& =\sum_{n=0}^{\infty} \sum_{j=0}^{n}\left(\begin{array}{l}
n \\
j
\end{array}\right) \sum_{k=0}^{j}(\alpha+k-1)_{k} S_{2}(j, k ; \lambda) y^{k} x^{n-j} \frac{t^{n}}{n !} .
\end{aligned}
$$

Comparing the coefficients of $\frac{\mathrm{t}^{\mathrm{n}}}{\mathrm{n} !}$ gives the desired result.

Theorem 5.2. For $\lambda \in \mathbb{C}$ and $\alpha, n \in \mathbb{N}_{0}$, we have

$$
x^{n}=\sum_{k=0}^{n}\left(\begin{array}{l}
n \\
k
\end{array}\right) \sum_{j=0}^{\alpha}(\alpha) j(-y)^{j} S_{2}(k, j ; \lambda) F_{n-k}^{(\alpha)}(x, y ; \lambda) .
$$

Proof. Expressing (2.1) into

$$
e^{x t}=\left(1-y\left(\lambda e^{t}-1\right)\right)^{\alpha} \sum_{n=0}^{\infty} F_{n}^{(\alpha)}(x, y ; \lambda) \frac{t^{n}}{n !},
$$

gives

$$
\begin{aligned}
\sum_{n=0}^{\infty} x^{n} \frac{t^{n}}{n !} & =\sum_{j=0}^{\alpha}\left(\begin{array}{l}
\alpha \\
j
\end{array}\right)(-y)^{j} j ! \frac{\left(\lambda e^{t}-1\right)^{j}}{j !} \cdot \sum_{n=0}^{\infty} F_{n}^{(\alpha)}(x, y ; \lambda) \frac{t^{n}}{n !} \\
& =\sum_{n=0}^{\infty} \sum_{j=0}^{\alpha}(\alpha)_{j}(-y)^{j} S_{2}(n, j ; \lambda) \frac{t^{n}}{n !} \cdot \sum_{n=0}^{\infty} F_{n}^{(\alpha)}(x, y ; \lambda) \frac{t^{n}}{n !} \\
& =\sum_{n=0}^{\infty}\left(\sum_{k=0}^{n}\left(\begin{array}{l}
n \\
k
\end{array}\right) \sum_{j=0}^{\alpha}(\alpha)_{j}(-y)^{j} S_{2}(k, j ; \lambda) F_{n-k}^{(\alpha)}(x, y ; \lambda)\right) \frac{t^{n}}{n !} .
\end{aligned}
$$

Theorem 5.3. For For $\lambda \in \mathbb{C}$ and $\alpha, n \in \mathbb{N}_{0}$, we have

$$
F_{n}^{(\alpha)}(x, y ; \lambda)=\sum_{k=0}^{\infty} \sum_{l=k}^{n}\left(\begin{array}{l}
n \\
l
\end{array}\right) F_{n-l}^{(\alpha)} S_{2}(l, k ; \lambda) \lambda^{-x-k} \chi^{(k)},
$$

where $x^{(0)}=1$, and $x^{(n)}=x(x+1)(x+2) \cdots(x+n-1), n \geqslant 1$.

Proof. Applying (2.1),

$$
\begin{aligned}
\sum_{n=0}^{\infty} F_{n}^{(\alpha)}(x, y, ; \lambda) \frac{t^{n}}{n !} & =\frac{1}{\left(1-y\left(\lambda e^{t}-1\right)\right)^{\alpha}}\left(\lambda-\left(\lambda-e^{-t}\right)\right)^{-x} \\
& =\frac{1}{\left(1-y\left(\lambda e^{t}-1\right)\right)^{\alpha}} \sum_{k=0}^{\infty}\left(\begin{array}{c}
x+k-1 \\
k
\end{array}\right) \lambda^{-x-k}\left(\lambda-e^{-t}\right)^{k} \\
& =\frac{1}{\left(1-y\left(\lambda e^{t}-1\right)\right)^{\alpha}} \sum_{k=0}^{\infty}\left(\begin{array}{c}
x+k-1 \\
k
\end{array}\right) \lambda^{-x-k} k ! \frac{\left(\lambda e^{t}-1\right)^{k}}{k !} e^{-k t} \\
& =\sum_{k=0}^{\infty} x^{(k)} \lambda^{-(x+k)} \sum_{n=0}^{\infty} F_{n}^{(\alpha)}(-k, y ; \lambda) \frac{t^{n}}{n !} \cdot \sum_{n=0}^{\infty} S_{2}(n, k ; \lambda) \frac{t^{n}}{n !}
\end{aligned}
$$




$$
\begin{aligned}
& =\sum_{k=0}^{\infty} x^{(k)} \lambda^{-(x+k)} \sum_{n=0}^{\infty} \sum_{l=0}^{n}\left(\begin{array}{c}
n \\
l
\end{array}\right) F_{n-l}^{(\alpha)}(-k, y ; \lambda) S_{2}(l, k ; \lambda) \frac{t^{n}}{n !} \\
& =\sum_{n=0}^{\infty}\left[\sum_{k=0}^{\infty} \sum_{l=k}^{n}\left(\begin{array}{c}
n \\
l
\end{array}\right) F_{n-l}^{(\alpha)} S_{2}(l, k ; \lambda) \lambda^{-x-k} x^{(k)}\right] \frac{t^{n}}{n !} .
\end{aligned}
$$

5.2. Relations involving Apostol-Fubini polynomials and other Apostol-type polynomials

Theorem 5.4. For $\alpha, \beta, \lambda \in \mathbb{C}$ and $n \in \mathbb{N}_{0}$, we have the following relationships:

$$
\begin{aligned}
& B_{n}^{(\alpha)}(x+z ; \lambda)=\sum_{k=0}^{n}\left(\begin{array}{l}
n \\
k
\end{array}\right) F_{k}^{(\beta)}(x, y ; \lambda)\left(\sum_{m \geqslant 0}\left(\begin{array}{c}
\beta \\
m
\end{array}\right)(y+1)^{\beta-m}(-y \lambda)^{m} B_{n-k}^{(\alpha)}(z+m ; \lambda)\right), \\
& E_{n}^{(\alpha)}(x+z ; \lambda)=\sum_{k=0}^{n}\left(\begin{array}{l}
n \\
k
\end{array}\right) F_{k}^{(\beta)}(x, y ; \lambda)\left(\sum_{m \geqslant 0}\left(\begin{array}{c}
\beta \\
m
\end{array}\right)(y+1)^{\beta-m}(-y \lambda)^{m} E_{n-k}^{(\alpha)}(z+m ; \lambda)\right), \\
& G_{n}^{(\alpha)}(x+z ; \lambda)=\sum_{k=0}^{n}\left(\begin{array}{l}
n \\
k
\end{array}\right) F_{k}^{(\beta)}(x, y ; \lambda)\left(\sum_{m \geqslant 0}\left(\begin{array}{c}
\beta \\
m
\end{array}\right)(y+1)^{\beta-m}(-y \lambda)^{m} G_{n-k}^{(\alpha)}(z+m ; \lambda)\right) .
\end{aligned}
$$

Proof. Note that

$$
\begin{aligned}
\left(\frac{t}{\lambda e^{t}-1}\right)^{\alpha} e^{(x+z) t} & =\frac{1}{\left(1-y\left(\lambda e^{t}-1\right)\right)^{\beta}} e^{x t} \cdot\left(\frac{t}{\lambda e^{t}-1}\right)^{\alpha} e^{z t}\left(1-y\left(\lambda e^{t}-1\right)\right)^{\beta} \\
& =\frac{1}{\left(1-y\left(\lambda e^{t}-1\right)\right)^{\beta}} e^{x t} \cdot\left(\frac{t}{\lambda e^{t}-1}\right)^{\alpha} e^{z t} \sum_{m \geqslant 0}\left(\begin{array}{c}
\beta \\
m
\end{array}\right)(y+1)^{\beta-m}(-y \lambda)^{m} e^{t m} .
\end{aligned}
$$

Thus,

$$
\begin{aligned}
\sum_{n=0}^{\infty} B_{n}^{(\alpha)}(x+z ; \lambda) \frac{t^{n}}{n !} & =\sum_{n=0}^{\infty} F_{n}^{(\beta)}(x, y ; \lambda) \frac{t^{n}}{n !} \cdot \sum_{m \geqslant 0}\left(\begin{array}{c}
\beta \\
m
\end{array}\right)(y+1)^{\beta-m}(-\lambda y)^{m} \sum_{n=0}^{\infty} B_{n}^{(\alpha)}(z+m ; \lambda) \frac{t^{n}}{n !} \\
& =\sum_{n=0}^{\infty}\left[\sum_{k=0}^{n}\left(\begin{array}{c}
n \\
k
\end{array}\right)\left(\sum_{m \geqslant 0}\left(\begin{array}{c}
\beta \\
m
\end{array}\right)(y+1)^{\beta-m}(-y \lambda)^{m} B_{k}^{(\alpha)}(z+m ; \lambda)\right) F_{n-k}^{(\beta)}(x, y ; \lambda)\right] \frac{t^{n}}{n !} .
\end{aligned}
$$

Comparing the coefficients of $\frac{t^{n}}{n !}$, we obtain (5.1). Relations (5.2) and (5.3) can be derived analogously.

Corollary 5.5. For $\alpha, \beta, \lambda \in \mathbb{C}$ and $n \in \mathbb{N}_{0}$, we have the following relationships:

$$
\begin{aligned}
& \mathrm{B}_{\mathrm{n}}^{(\alpha)}(x+z ; \lambda)=\sum_{k=0}^{n}\left(\begin{array}{l}
n \\
k
\end{array}\right)\left[\mathrm{B}_{k}^{(\alpha)}(z ; \lambda)-y k B_{k-1}^{(\alpha-1)}(z ; \lambda)\right] F_{n-k}(x, y ; \lambda), \\
& E_{n}^{(\alpha)}(x+z ; \lambda)=\sum_{k=0}^{n}\left(\begin{array}{l}
n \\
k
\end{array}\right)\left[(2 y+1) E_{k}^{(\alpha)}(z ; \lambda)-2 y E_{k}^{(\alpha-1)}(z ; \lambda)\right] F_{n-k}(x, y ; \lambda), \\
& G_{n}^{(\alpha)}(x+z ; \lambda)=\sum_{k=0}^{n}\left(\begin{array}{l}
n \\
k
\end{array}\right)\left[(2 y+1) G_{k}^{(\alpha)}(z ; \lambda)-2 y k G_{k-1}^{(\alpha-1)}(z ; \lambda)\right] F_{n-k}(x, y ; \lambda) .
\end{aligned}
$$

Proof. Setting $\beta=1$ in Theorem 5.4, we obtain

$$
\mathrm{B}_{n}^{(\alpha)}(x+z ; \lambda)=\sum_{k=0}^{n}\left(\begin{array}{l}
n \\
k
\end{array}\right)\left[(y+1) B_{k}^{(\alpha)}(z ; \lambda)-y \lambda B_{k}^{(\alpha)}(z+1 ; \lambda)\right] F_{n-k}(x, y ; \lambda),
$$




$$
\begin{aligned}
& E_{n}^{(\alpha)}(x+z ; \lambda)=\sum_{k=0}^{n}\left(\begin{array}{l}
n \\
k
\end{array}\right)\left[(y+1) E_{k}^{(\alpha)}(z ; \lambda)-y \lambda E_{k}^{(\alpha)}(z+1 ; \lambda)\right] F_{n-k}(x, y ; \lambda), \\
& G_{n}^{(\alpha)}(x+z ; \lambda)=\sum_{k=0}^{n}\left(\begin{array}{l}
n \\
k
\end{array}\right)\left[(y+1) G_{k}^{(\alpha)}(z ; \lambda)-y \lambda G_{k}^{(\alpha)}(z+1 ; \lambda)\right] F_{n-k}(x, y ; \lambda) .
\end{aligned}
$$

Using the recurrence relations (1.7), (1.8), and (1.9), we obtain the desired identities.

Next, by setting $\lambda=1$ in Theorem 5.4 , we obtain the following identities involving higher order Apostol-type polynomials and higher order bivariate Fubini polynomials.

Corollary 5.6. For $\alpha, \beta, \in \mathbb{C}$ and $n \in \mathbb{N}_{0}$, we have the following relationships:

$$
\begin{aligned}
& B_{n}^{(\alpha)}(x+z)=\sum_{k=0}^{n}\left(\begin{array}{l}
n \\
k
\end{array}\right)\left(\sum_{m \geqslant 0}\left(\begin{array}{l}
\beta \\
m
\end{array}\right)(y+1)^{\beta-m}(-y)^{m} B_{n-k}^{(\alpha)}(z+m)\right) F_{k}^{(\beta)}(x, y), \\
& E_{n}^{(\alpha)}(x+z)=\sum_{k=0}^{n}\left(\begin{array}{l}
n \\
k
\end{array}\right)\left(\sum_{m \geqslant 0}\left(\begin{array}{c}
\beta \\
m
\end{array}\right)(y+1)^{\beta-m}(-y)^{m} E_{n-k}^{(\alpha)}(z+m)\right) F_{k}^{(\beta)}(x, y), \\
& G_{n}^{(\alpha)}(x+z)=\sum_{k=0}^{n}\left(\begin{array}{l}
n \\
k
\end{array}\right)\left(\sum_{m \geqslant 0}\left(\begin{array}{c}
\beta \\
m
\end{array}\right)(y+1)^{\beta-m}(-y)^{m} G_{n-k}^{(\alpha)}(z+m)\right) F_{k}^{(\beta)}(x, y) .
\end{aligned}
$$

The next corollary gives an identity involving binomial expansion in terms of the bivariate ApostolFubini polynomials of higher order.

Corollary 5.7. For $\beta, \lambda \in \mathbb{C}$ and $n \in \mathbb{N}_{0}$, we have

$$
\begin{aligned}
(x+z)^{n} & =\sum_{m \geqslant 0}\left(\begin{array}{c}
\beta \\
m
\end{array}\right)(y+1)^{\beta-m}(-y \lambda)^{m} F_{k}^{(\beta)}(x+z+m, y ; \lambda), \\
x^{n} & =\sum_{m \geqslant 0}\left(\begin{array}{c}
\beta \\
m
\end{array}\right)(y+1)^{\beta-m}(-y \lambda)^{m} F_{k}^{(\beta)}(x+m, y ; \lambda) .
\end{aligned}
$$

Proof. Setting $\alpha=0$ in Theorem 5.4, and then applying Theorem 2.4, we obtain

$$
\begin{aligned}
(x+z)^{n} & =\sum_{m \geqslant 0}\left(\begin{array}{c}
\beta \\
m
\end{array}\right)(y+1)^{\beta-m}(-y \lambda)^{m} \sum_{k=0}^{n}\left(\begin{array}{l}
n \\
k
\end{array}\right) F_{k}^{(\beta)}(x, y ; \lambda)(z+m)^{n-k} \\
& =\sum_{m \geqslant 0}\left(\begin{array}{c}
\beta \\
m
\end{array}\right)(y+1)^{\beta-m}(-y \lambda)^{m} F_{n}^{(\beta)}(x+z+m, y ; \lambda) .
\end{aligned}
$$

Taking $z=0$ in (5.4), we obtain (5.5).

In the next result, we express $F_{n}^{(\alpha)}(x, y ; \lambda)$ in terms of the higher order Apostol-Euler polynomials.

Theorem 5.8. For $\alpha, \beta, \lambda \in \mathbb{C}$ and $n \in \mathbb{N}_{0}$, we have

$$
F_{n}^{(\alpha)}(x+z, y ; \lambda)=\frac{1}{2^{\beta}} \sum_{k=0}^{n}\left(\begin{array}{l}
n \\
k
\end{array}\right) \sum_{m \geqslant 0}\left(\begin{array}{l}
\beta \\
m
\end{array}\right) \lambda^{m} F_{n-k}^{(\alpha)}(z+m, y ; \lambda) E_{k}^{(\beta)}(x ; \lambda) .
$$


Proof. Note that

$$
\frac{e^{(x+z) t}}{\left(1-y\left(\lambda e^{t}-1\right)\right)^{\alpha}}=\frac{e^{z t}}{\left(1-y\left(\lambda e^{t}-1\right)\right)^{\alpha}}\left(\frac{2}{\lambda e^{t}+1}\right)^{\beta} e^{x t} \cdot \frac{1}{2^{\beta}}\left(\lambda e^{t}-1\right)^{\beta}
$$

Hence,

$$
\begin{aligned}
\sum_{n=0}^{\infty} F_{n}^{(\alpha)}(x+z, y ; \lambda) \frac{t^{n}}{n !} & =\frac{e^{z t}}{\left(1-y\left(\lambda e^{t}-1\right)\right)^{\alpha}} \cdot\left(\frac{2}{\lambda e^{t}+1}\right)^{\beta} e^{x t} \frac{1}{2^{\beta}} \sum_{m \geqslant 0}\left(\begin{array}{c}
\beta \\
m
\end{array}\right) \lambda^{m} e^{t m} \\
& =\sum_{n=0}^{\infty}\left[\frac{1}{2^{\beta}} \sum_{k=0}^{n}\left(\begin{array}{l}
n \\
k
\end{array}\right) \sum_{m \geqslant 0}\left(\begin{array}{c}
\beta \\
m
\end{array}\right) \lambda^{m} F_{n-k}^{(\alpha)}(z+m, y ; \lambda) E_{k}^{(\alpha)}(x ; \lambda)\right] \frac{t^{n}}{n !} .
\end{aligned}
$$

Comparing the coefficients of $\frac{\mathrm{t}^{\mathrm{n}}}{\mathrm{n} !}$ gives the result.

Corollary 5.9. For $\alpha, \lambda \in \mathbb{C}$ and $n \in \mathbb{N}_{0}$, we have

$$
\begin{aligned}
F_{n}^{(\alpha)}(x+z, y ; \lambda) & =\frac{1}{2 y} \sum_{k=0}^{n}\left(\begin{array}{l}
n \\
k
\end{array}\right)\left[(2 y+1) F_{k}^{(\alpha)}(x, y ; \lambda)-F_{k}^{(\alpha-1)}(z, y ; \lambda)\right] E_{n-k}^{(\beta)}(x ; \lambda), \\
F_{n}^{(\alpha)}(x+z, y) & =\frac{1}{2^{\beta}} \sum_{k=0}^{n}\left(\begin{array}{l}
n \\
k
\end{array}\right) \sum_{m \geqslant 0}\left(\begin{array}{l}
\beta \\
m
\end{array}\right) F_{n-k}^{(\alpha)}(z+m, y) E_{k}^{(\beta)}(x) .
\end{aligned}
$$

Proof. Taking $\beta=1$ in Theorem 5.8, we obtain

$$
\left.F_{n}^{(\alpha)}(x+z, y ; \lambda)=\frac{1}{2} \sum_{k=0}^{n}\left(\begin{array}{l}
n \\
k
\end{array}\right)\left(F_{n-k}^{(\alpha)}(z, y ; \lambda)+\lambda F_{n-k}^{(\alpha)}(z+1), y ; \lambda\right)\right) E_{k}^{(\alpha)}(x ; \lambda) .
$$

and then applying recurrence relation (2.2), we get (5.6). Now, by setting $\lambda=1$ in Theorem 5.8, relation (5.7) is obtained.

Taking $\alpha=0$ in Corollary 5.9, we obtain expressions involving the binomial expansion.

Corollary 5.10. For $\beta, \lambda \in \mathbb{C}$ and $n \in \mathbb{N}_{0}$, we have

$$
\begin{aligned}
& (x+z)^{n}=\frac{1}{2 y} \sum_{k=0}^{n}\left(\begin{array}{l}
n \\
k
\end{array}\right)\left[(2 y+1) x^{k}-F_{k}^{(-1)}(z, y ; \lambda)\right] E_{n-k}^{(\beta)}(x ; \lambda), \\
& (x+z)^{n}=\frac{1}{2^{\beta}} \sum_{m \geqslant 0}\left(\begin{array}{l}
\beta \\
m
\end{array}\right) E_{n}^{(\beta)}(x+z+m) .
\end{aligned}
$$

\section{Acknowledgment}

The author greatly appreciates the reviewers for their valuable comments and suggestions for this paper.

\section{References}

[1] N. G. Acala, A unification of the generalized multiparameter Apostol-type Bernoulli, Euler, Fubini, and Genocchi polynomials of higher order, Eur. J. Pure Appl. Math., 13 (2020), 587-607. 2.1

[2] S. Araci, W. A. Khan, M. Acikgoz, C. Ozel, P. Kumam, A new generalization of Apostol-type Hermite-Genocchi polynomials and its application, SpringerPlus, 5 (2016), 17 pages. 1 
[3] R. Dere, Y. Simsek, Hermite base Bernoulli type polynomials on the umbral algebra, Russ. J. Math. Phys., 22 (2015), 1-5. 2.2

[4] R. Dere, Y. Simsek, H. M. Srivastava, A unified presentation of three families of generalized Apostol type polynomials based upon the theory of the umbral calculus and the umbral algebra, J. Number Theory, 133 (2013), 3245-3263. 1

[5] A. Dil, V. Kurt, Investigating geometric and exponential polynomials with Euler-Seidel matrices, J. Integer Seq., 14 (2011), 12 pages. 1

[6] U. Duran, S. Araci, M. Acikgoz, A note on q-Fubini polynomials, Adv. Stud. Contemp. Math. (Kyungshang), 29 (2019), 211-224. 1

[7] R. L. Graham, D. E. Knuth, O. Patashnik, Concrete Mathematics, Addison-Wesley Publ. Co., New York, (1994). 1

[8] G.-W. Jang, T. Kim, Some identities of the ordered Bell numbers arising from differential equations, Adv. Stud. Contemp. Math. (Kyungshang), 27 (2017), 385-397. 1

[9] L. Kargin, Some formulae for products of geometric polynomials with applications, J. Integer Seq., 20 (2017), 15 pages. 1.3

[10] N. Kilar, Y. Simsek, A new family of Fubini type numbers and polynomials associated with Apostol-Bernoulli numbers and polynomials, J. Korean Math. Soc., 54 (2017), 1605-1621. 1.3

[11] T. Kim, Degenerate ordered Bell numbers and polynomials, Proc. Jangjeon Math. Soc., 20 (2017), 137-144. 1

[12] D. S. Kim, G.-W. Jang, H.-I. Kwon, T. Kim, Two variable higher-order degenerate Fubini polynomials, Proc. Jangjeon Math. Soc., 21 (2018), 5-22. 1.2

[13] T. Kim, D. S. Kim, G.-W. Jang, A note on degenerate Fubini polynomials, Proc. Jangjeon Math. Soc., 20 (2017), $521-531$. 1.3

[14] T. Kim, D. S. Kim, G.-W. Jang, D. Kim, Two variable higher-order central Fubini polynomials, J. Inequal. Appl., (2019), 13 pages. 1.2

[15] T. Kim, D. S. Kim, G.-W. Jang, J. Kwon, Symmetric identities for Fubini polynomials, Symmetry, 10 (2018), 7 pages.

[16] D. S. Kim, T. Kim, H.-I. Kwon, J.-W. Park, Two variable higher-order Fubini polynomials, J. Korean Math. Soc., 55 (2018), 975-986. 1.2

[17] V. Kurt, A further symmetric relation on the analogue of the Apostol-Bernoulli and the analogue of the Apostol-Genocchi polynomials, Appl. Math. Sci., 3 (2009), 2757-2764. 1

[18] Q.-M. Luo, On the Apostol-Bernoulli polynomials, Cent. Eur. J. Math., 2 (2004), 509-515.

[19] Q.-M. Luo, Apostol-Euler polynomials of higher order and Gaussian hypergeometric functions, Taiwanese J. Math., 10 (2006), 917-925.

[20] Q.-M. Luo, H. M. Srivastava, Some generalizations of the Apostol-Bernoulli and Apostol-Euler polynomials, J. Math. Anal. Appl., 308 (2005), 290-302.

[21] Q.-M. Luo, H. M. Srivastava, Some generalizations of the Apostol-Genocchi polynomials and the Stirling numbers of the second kind, Appl. Math. Comput., 217 (2011), 5702-5728. 1, 2.2, 3

[22] D. Q. Lu, H. M. Srivastava, Some series identities involving the generalized Apostol type and related polynomials, Comput. Math. Appl., 62 (2011), 3591-3602. 4

[23] H. M. Srivastava, Some generalizations and basic (or q-)extensions of the Bernoulli, Euler and Genocchi polynomials, Appl. Math. Inform. Sci., 5 (2011), 390-444. 2.2

[24] H. M. Srivastava, P. G. Todorov, An explicit formula of the generalized Bernoulli polynomials, J. Math. Anal. Appl., 130 (1988), 509-513. 1

[25] S. M. Tanny, On some numbers related to the Bell numbers, Canad. Math. Bull., 17 (1974/75), 733-738. 1

[26] W. Wang, C. Jia, T. Wang, Some results on the Apostol-Bernoulli and Apostol-Euler polynomials, Comput. Math. Appl., 55 (2008), 1322-1332. 1

[27] Z. Zhang, H. Yang, Several identities for the generalized Apostol-Bernoulli polynomials, Comput. Math. Appl., 56 (2008), 2993-2999. 1 